\title{
Fatal Lemierre's syndrome as a complication of chronic otitis media with cholesteatoma
}

\author{
M Roos, ${ }^{1,2}$ MB ChB; T Harris, ${ }^{1,3}$ MB ChB, FCORL(SA), MMed (Otol); R Seedat, ${ }^{2}$ MB ChB, MMed (ORL), FCORL(SA) \\ ${ }^{1}$ Department of Otolaryngology, Kimberley Hospital Complex, South Africa \\ ${ }^{2}$ Department of Otorhinolaryngology, Faculty of Health Sciences, University of the Free State, Bloemfontein, South Africa \\ ${ }^{3}$ Division of Otolaryngology, Department of Surgery, Faculty of Health Sciences, University of Cape Town, South Africa
}

Corresponding author: $M$ Roos (visagieme@gmail.com)

\begin{abstract}
Background. Lemierre's syndrome is septic thrombophlebitis of the internal jugular vein, initiated by an infection of the head and neck region. This septic thrombophlebitis gives rise to septic microemboli that can disseminate throughout the body to form septic infarctions and abscesses, with the most frequent site being pulmonary.

Methods. We discuss the case of a 14-year-old male with Lemierre's syndrome as a complication of chronic middle ear infection.

Results. The patient developed septic shock and microemboli, and subsequently died.

Conclusion. This case report illustrates that untreated chronic middle ear infection can lead to potentially fatal complications such as Lemierre's syndrome, and emphasises the importance of timeous treatment of chronic middle ear pathology.

S Afr J Child Health 2016; 10(4):231-232. DOI:10.7196/SAJCH.2016.v10i4.1074
\end{abstract}

Lemierre's syndrome is septic thrombophlebitis of the internal jugular vein (IJV) occurring secondary to infection in the head and neck region of otherwise healthy young patients. ${ }^{[1]}$ The thrombophlebitis is often followed by pulmonary emboli and distal septic embolisation. ${ }^{[2,3]}$ The syndrome was first described in 1936 as sepsis secondary to Fuscobacterium necrophorum, although other organisms, for example Streptococcus, Staphylococcus, Lactobacillus, Bacteroides, Peptostreptococcus and Eikenella, are also described as being involved in the aetiology of this syndrome..$^{[1,2]}$ Although it is an uncommon syndrome (it is also referred to as the 'forgotten disease'), a high index of suspicion should be maintained, as it remains a potentially life-threatening disease with a reported mortality rate of up to $10 \% \cdot{ }^{[3]} \mathrm{A}$ few cases of Lemierre's syndrome have been reported with the primary event being otological in nature. ${ }^{[1,3,4]}$ The following report describes the fatal outcome of a 14-year-old boy who developed Lemierre's syndrome secondary to untreated chronic otitis media with cholesteatoma.

\section{Case report}

A previously healthy 14-year-old boy was admitted to hospital with a 1-week history of worsening headaches, photophobia and malaise. The patient had been seen in casualty 3 days prior to admission, diagnosed with an acute otitis media and treated with oral antibiotics. There was a history of right-sided otorrhoea for many years and, despite multiple visits to his local clinic, the patient was never referred to an otolaryngologist. There was no other relevant past medical or surgical history.

On admission, the patient was pyrexial with a temperature of $38.2^{\circ} \mathrm{C}$ and had rigors. His blood pressure was $114 / 44 \mathrm{mmHg}$ and his pulse $62 \mathrm{bpm}$. The respiratory and cardiovascular examinations were normal. The patient was noted to have a discharging right ear and tenderness of his neck. There were no signs of meningeal irritation. The results of the laboratory investigations were as follows: white cell count of $28.8 \times 10^{9} / \mathrm{L}$, haemoglobin of $9.5 \mathrm{~g} / \mathrm{dL}$, platelets of $120 \times 10^{9} / \mathrm{L}$ and a C-reactive protein level of $190.8 \mathrm{mg} / \mathrm{L}$. A lumbar puncture was performed to exclude meningitis and this was normal. The chest X-ray (CXR) on admission revealed no abnormalities. Empirical treatment was started with intravenous ceftriaxone $2 \mathrm{~g}$ twice daily. Aerobic and anaerobic cultures were taken, with negative results.

After 1 week, there was no clinical improvement and the signs of sepsis persisted. The intravenous antibiotics were changed to gentamicin $240 \mathrm{mg}$ once daily and ertapenem $1 \mathrm{~g}$ daily. The patient then complained of central chest pain and shortness of breath. His blood pressure was $118 / 69 \mathrm{mmHg}$ and his respiratory rate was 28 breaths/min. An electrocardiogram was done, showing a sinus tachycardia. A repeat lumbar puncture revealed no abnormalities and blood cultures remained negative. Intravenous vancomycin $1 \mathrm{~g}$ daily was added to the treatment.

A repeat CXR showed small focal opacities in both lung fields consistent with septic emboli. An abdominal ultrasound was done, revealing hepatosplenomegaly and a solitary cystic lesion of $3 \mathrm{~cm} \times 3 \mathrm{~cm}$ in the liver. An urgent computed tomography (CT) scan of the brain and temporal bones was requested, revealing an opacified middle ear and epitympanum. An extensive thrombus was seen in the right transverse dural sinus (Fig. 1), extending inferiorly into the sigmoid sinus and IJV. Dehiscence of the posterior cerebellar plate was seen, but there was no intracranial collection.

The patient was referred to the ear, nose and throat (ENT) department for further assessment and management. On ENT examination, the presence of keratin and a granuloma was noted in the right ear and the diagnosis of a right-sided cholesteatoma was made. A pus swab taken from the right ear cultured Proteus vulgaris and Pseudomonas aeruginosa. Audiological testing revealed a mild conductive hearing loss of the right ear, but normal hearing in the left.

The patient developed haemoptysis and deteriorated clinically, secondary to a suspected pulmonary embolism and septic shock. He was resuscitated and transferred to the intensive care unit. A repeat heart sonar revealed a normal right heart, tachycardia of 141 beats/ min and no signs of a pulmonary embolism. A repeat CXR showed worsening of the opacification, consistent with pneumonia.

$\mathrm{He}$ was started on enoxaparin $40 \mathrm{mg}$ 12-hourly subcutaneously. $\mathrm{He}$ developed a right-sided pneumothorax and an intercostal chest drain was inserted. His sepsis continued and he required inotropic support. He also developed generalised tonic-clonic seizures and was started on intravenous phenytoin. He had repeated cardiac arrests, and 


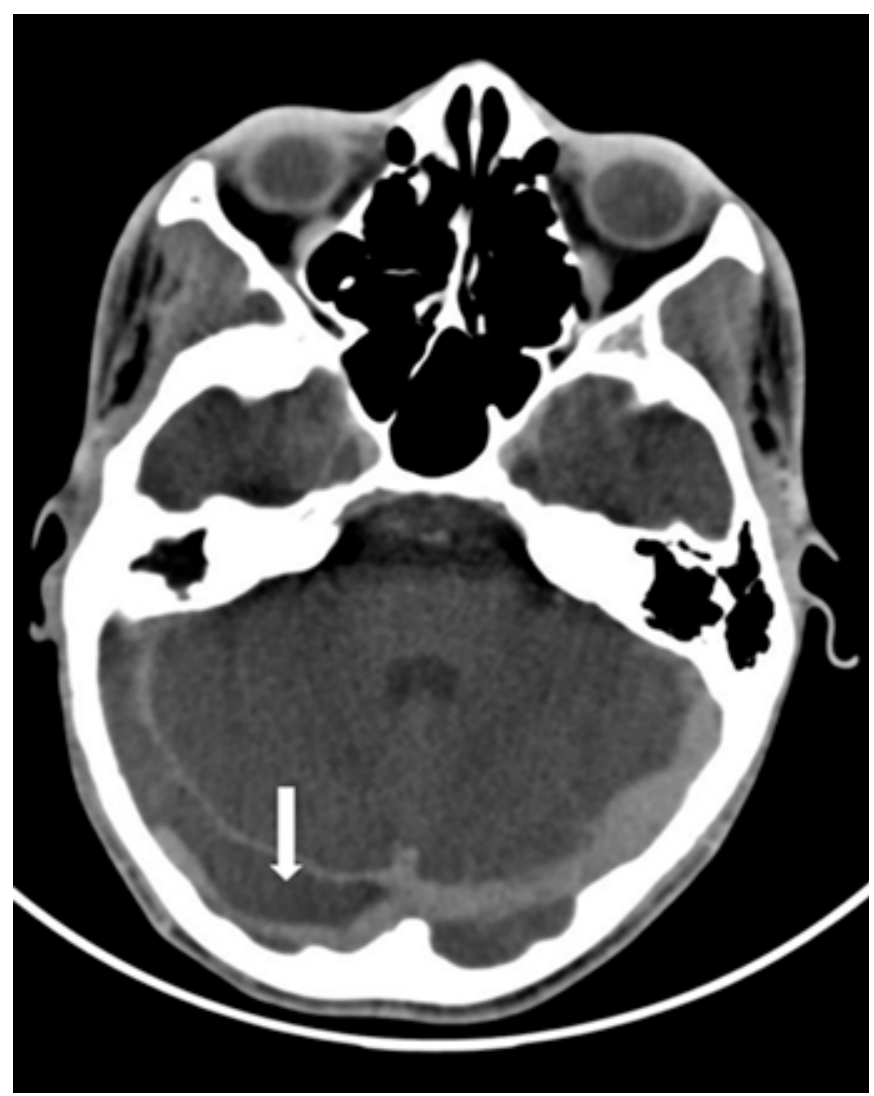

Fig. 1. Post-contrast CT brain showing filling defect in the right transverse and sigmoid sinus with the appearance of a thrombus.

after 2 days in intensive care it was noted that he had no brainstem reflexes. After 4 days in intensive care all active resuscitative efforts were stopped and the patient died shortly thereafter.

\section{Discussion}

The clinical presentation of Lemierre's syndrome includes fever, neck swelling, respiratory symptoms, oropharyngeal pain, hepatosplenomegaly, jaundice, haematuria, diffuse encephalopathy, myalgia and arthralgia. ${ }^{[1,2]}$ Owing to the wide range of presenting symptoms and signs, the diagnosis could easily be missed if not actively sought. Findings on CXR may include infiltrates, embolic phenomena, pleural effusion, pneumothorax and empyema, but a normal CXR does not exclude the diagnosis. ${ }^{[2,3]}$ The incidence ranges from 0.6 to 2.3 cases per million per year, ${ }^{[4]}$ although progressively more cases have been reported, possibly because of the development of antibiotic resistance. ${ }^{[1]}$

Complications of Lemierre's syndrome include extension of the thrombophlebitis to involve other vessels such as the pharyngeal venous plexus and cavernous sinus, meningitis, brain abscesses, descending necrotising mediastinitis, necrosis of infected blood vessels, suppurative arthritis and endocarditis. ${ }^{[1]}$ With otogenic infection the close proximity of the middle ear and mastoid to the dural venous sinuses predispose these regions to lateral sinus thrombophlebitis. Formation may be either a direct dissemination through neighbouring eroded bone or from thrombophlebitis of small veins of the mastoid communicating with the lateral sinus. ${ }^{[3]}$ Extension of the thrombus may be significant, reaching the superior petrosal sinus superior, or as low as the subclavian vein. ${ }^{[3]}$ Another complication is intravascular coagulation secondary to platelet aggregation, possibly contributing to the thrombophlebitis, by creating a favourable environment for growth of anaerobic bacteria. ${ }^{[4]}$ This was seen in our patient, who developed sepsis-related thrombocytopenia with a platelet count of $45 \times 10^{9} / \mathrm{L}$.

The septic thrombophlebitis of the IJV may lead to metastatic septic emboli with pulmonary involvement in up to $85 \%$ of cases. ${ }^{[1]}$ Distal complications may be present in about $90 \%$ of cases at the time of diagnosis. ${ }^{[4]}$ We suspected that the probable cause of the patient's ongoing sepsis was septic microemboli to the lungs, although the heart sonar ruled out a large pulmonary embolus. He was therefore started on enoxaparin. The use of anticoagulation is controversial, as there are no randomised control trials and septis-related thrombocytopenia is often an associated complication, possibly increasing the risk of bleeding. ${ }^{[2,3]}$

Antibiotics are the mainstay of treatment of Lemierre's syndrome ${ }^{[1,2]}$ and should be continued for at least 6 weeks. ${ }^{[2]}$ Combined treatment with penicillin and metronidazole are recommended. ${ }^{[3]}$ It is crucial to take blood cultures early to allow initiation of the appropriate therapy. The patient's blood cultures remained negative, possibly because antibiotics were initiated at the time the first cultures were requested. In children with chronic suppurative otitis media, Gramnegative bacteria are the most common pathogens cultured, with a high sensitivity to quinolones. ${ }^{[5]}$

Surgical excision and ligation of the IJV is reserved for cases with continued sepsis and septic embolisation. ${ }^{[3]}$ Further management includes surgical drainage of any purulent collections. ${ }^{[3]}$ Contrastenhanced CT is the imaging modality of choice for accurate diagnosis of Lemierre's syndrome. Ultrasonography has been used as an initial investigation; however, it can miss a fresh thrombus. Magnetic resonance imaging has also been suggested as the study of choice and has the added advantage of avoiding an intravenous contrast agent and exposure to radiation. ${ }^{[3]}$

The delay in diagnosis of chronic suppurative otitis media is a significant problem. Tiedt et al. ${ }^{[5]}$ noted a long delay in seeking treatment for chronic middle ear infection, with the mean duration of otorrhoea being $>3$ years. It is important to have a high index of suspicion as patients who are diagnosed and treated early are reported to have a favourable outcome. ${ }^{[2]}$ Patients with chronic middle ear infection often have associated comorbidities such as anaemia, malnutrition and HIV infection. ${ }^{[6]}$

\section{Conclusion}

Lemierre's syndrome is a rare but life-threatening complication of untreated middle ear infections. This case study demonstrates the importance of treating any middle ear infection timeously as it could be fatal if left untreated.

\section{References}

1. Gilbert JD, Warner MS, Byard RW. Lemierre syndrome and unexpected death in childhood. J Forensic Leg Med 2009;16(8):478-481. http://dx.doi. org/10.1016/j.jflm.2009.07.005

2. Chen $\mathrm{CH}$, Lin $\mathrm{CH}$, Chin $\mathrm{CH}$, Chiu $\mathrm{MH}$, Chen PH. Lemierre's syndrome. J Med Ultrasound 2005;13(3):149-152.

3. Adhikari P, Guragain RP, Bhusal, CL, Pradhananga RB. Lemierre's syndrome after modified radical mastoidectomy: An unusual variant caused by Citrobacter freneli. Int J Pediatr Otorhinolaryngol Extra 2010;5(4):152-154. http://dx.doi. org/10.1016/j.ijporl.2004.07.023

4. Masterson T, El-Hakim H, Magnus K, Robinson J. A case of the otogenic variant of Lemierre's syndrome with atypical sequelae and review of pediatric literature. Int J Pediatr Otorhinolaryngol 2005;69(1):117-122. http://dx.doi.org/10.1016/j. ijporl.2004.07.023

5. Tiedt NJ, Butler IR, Hallbauer UM, et al. Paediatric chronic suppurative otitis media in the Free State Province: Clinical and audiological features. S Afr Med J 2013;103(7):467-470. http://dx.doi.org/10.7196/samj.6636

6. Hallbauer UM, Atkins MD, Tiedt NJ, et al. Co-morbidities in children presenting with chronic suppurative otitis media - A South African study. J Trop Pediatr 2014;60(3):198-202. http://dx.doi.org/10.1093/tropej/fmt107 